Document downloaded from:

http://hdl.handle.net/10251/70306

This paper must be cited as:

Grillo Espinoza, H.; Peidro Payá, D.; Alemany Díaz, MDM.; Mula, J. (2015). Application of particle swarm optimisation with backward calculation to solve a fuzzy multi-objective supply chain master planning model. International Journal of Bio-Inspired Computation. 7(3):157169. doi:10.1504/IJBIC.2015.069557.

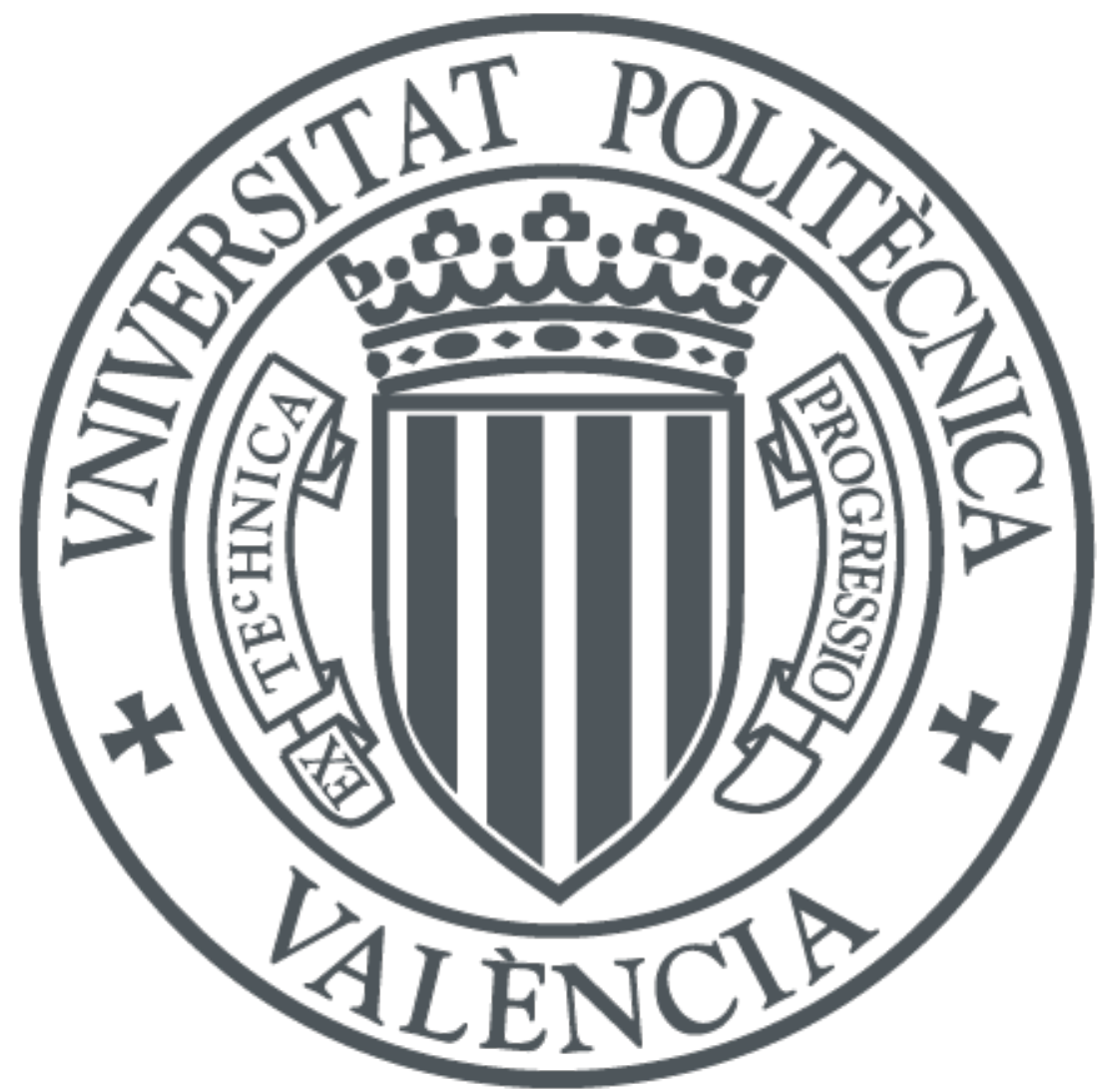

The final publication is available at

http://dx.doi.org/10.1504/IJBIC.2015.069557

Copyright Inderscience

Additional Information 


\title{
Application of Particle SWarm Optimization With BACKWARD CALCUlation (PSO-B) TO SOLVE A FUZZY Multiobjective Supply Chain Master Planning Model
}

\author{
Hanzel Grillo ${ }^{\mathrm{a}, *}$, David Peidróa, M.M.E Alemany ${ }^{\mathrm{a}}$, Josefa Mula ${ }^{\mathrm{a}}$. \\ ${ }^{a}$ Research Centre on Production Management and Engineering (CIGIP), Universitat Politècnica de \\ València, Camino de Vera s/n, 46022 Valencia, Spain.
}

\begin{abstract}
:
Traditionally, supply chain planning problems consider variables with uncertainty associated with uncontrolled factors. These factors have been normally modelled by complex methodologies where the seeking solution process often presents high scale of difficulty. This work presents the fuzzy set theory as a tool to model uncertainty in supply chain planning problems and proposes the particle swarm optimization (PSO) metaheuristics technique combined with a backward calculation as a solution method. The aim of this combination is to present a simple effective method to model uncertainty, while good quality solutions are obtained with metaheuristics due to its capacity to find them with satisfactory computational performance in complex problems, in a relatively short time period.
\end{abstract}

Keywords: Metaheuristics, particle swarm optimization, backward calculation, fuzzy sets, master planning, supply chain.

\section{Introduction}

Supply chain planning problems often involve variables or parameters affected by uncertainty conditions due to uncontrolled factors. This uncertainty is seen, for example, in demand conditions, production times, replenishment or distribution, types of customers, planned income and costs, among others. Traditionally, presence of uncertainty in such models has been considered by applying techniques like stochastic programming or simulation. Such methodologies entail the drawback of requiring considerable amounts of data or historic records of the variables affected by uncertainty to be able to establish probability distributions that describe their performance, which is not often feasible. Thus, what tends to happen is that uncertainty is not often modeled satisfactorily and realistically.

Furthermore, these mathematical problems have the peculiarity of depending on the scope and size of the supply chain, especially its characteristics, that they model, which can reach considerable solution complexity levels that make it difficult to find solutions of good quality or with poorly efficient computational performance. Thus highly complex solution models tend to be difficult to solve by conventional optimization mechanisms such as Simplex or differential optimization algorithms, used to seek optimum solutions, and developed in languages such as C++ or Java, among others.

Along these lines, this paper continues previous research on the centralized master planning of multiitem, multi-supplier, multi-facility, multi-type and multi-level distribution centers ceramic supply chains. This problem was originally considered in a deterministic environment by Alemany et al. (2010), through a mixed integer linear programming model, and subsequent modeling under uncertainty by applying the

\footnotetext{
* Corresponding author.

Research Centre on Production Management and Engineering (CIGIP)

Polytechnic University of Valencia

Camino de Vera S/N, 46023, Valencia, (SPAIN).

Tel.:+34 963877007 ext. 88484

Fax: +34963877689

E-mail address: hangries@upvnet.upv.es, grillo7@gmail.com
} 
fuzzy set theory by Peidro et al. (2012) resulting in the FMOLP (fuzzy multi objective linear programming) model. These models belong to the capacitated lot sizing and loading problem (CLSLP) with backlogging and setup carry-over category. Karimi et al. (2003) stated that there has been little literature regarding the above kind of CLSLP problems and since these problems are NP-hard, fast and efficient heuristics are required. For the purpose of contributing to this under-researched topic, the particle swarm optimization (PSO) metaheuristics technique has been selected to solve the FMOLP.

The PSO method was first introduced by Kennedy and Eberhart (1995). PSO is a bio-inspired algorithm based on artificial life (A-life) in general, and on bird flocking, fish schooling, and swarming theory in particular. It is also related, however, to evolutionary computation, and has ties to both genetic algorithms and evolutionary programming. Several examples exist in the literature that show that PSO is an efficient method to search for better solutions for hard problems. PSO has been proved to be an efficient method for many global optimization problems and in some cases it does not suffer the difficulties encountered by other evolutionary computation techniques (Kennedy and Eberhart, 1995). However, as it is typical with all the evolutionary algorithms, the PSO does not guarantee to get an optimal solution for a problem. Therefore, it is a good option for problems where obtaining an optimal solution is very difficult like in the problem presented in this paper.

In the field of supply chain, PSO has been applied in a different ways. Most of the papers encountered in the literature about the application of PSO in supply chain planning, are related to inventory management. Sadeghi et al. $(2013,2014)$ proposed a multi-vendor multi-retailer single-warehouse vendor managed inventory (VMI) policy formulated into an integer nonlinear programming model, the meta-heuristic algorithm of PSO is presented to find an approximate optimum solution of the problem. Sue-Ann et al. (2012) focused on the operational issues of a two-echelon single-vendor-multiple-buyers supply chain under VMI mode of operation. The operational parameters considered are sales quantity and sales price that determine the channel profit of the supply chain. The model is solved applying PSO and a hybrid of genetic algorithm and artificial immune system (GA-AIS). Taleizadeh et al. (2010) considered a single vendor-single buyer inventory problem in which the demand is stochastic and the lead time is assumed to vary linearly with respect to the lot size. Taleizadeh et al. (2012) expanded their previous work to a multiproduct multi-chance constraint joint single-vendor multi-buyers inventory problem. Finally in Taleizadeh et al. (2013) a chance-constraint supply chain problem with stochastic demand and fuzzy delay time is proposed. All the models are shown to be integer nonlinear programming type and in order to solve them a PSO approach is used jointly with other techniques like genetic algorithm (GA) and artificial bee colony. Wong et al. (2009) proposed a replenishment decision support system based on response surface methodology (RSM) and modified traveling particle swarm optimization (TPSO). This paper solved a two stage stochastic dynamic lot sizing problem with two phased transportation cost under a VMI. The solution quality using modified TPSO was tested and compared with that of the Solver tool in Excel and 3 lot sizing decision rules. In Mousavi et al. (2014) a seasonal multi-product multi-period inventory control problem is modeled. The objective is to find the optimal number of boxes of the products in different periods to minimize the total inventory cost (including ordering, holding, shortage, and purchasing costs). Since the integer nonlinear model of the problem is hard to solve using exact methods, a PSO algorithm is proposed to find a near-optimal solution and is compared with the application of a GA. Park and Kyung (2014) proposed a method to optimize both the total cost and order fill rates in a supply chain using the PSO method. Yang and Lin (2010) provided a serial multi-echelon integrated just-in-time (JIT) model based on uncertain delivery lead time and quality unreliability considerations. They applied PSO as a method to result an improved solution solving a mixed nonlinear integer problem. Yaghin et al. (2013) considered pricing, marketing and lot-sizing decisions simultaneously, presented a fuzzy non-linear multiobjective model that maximizes the profit and return on inventory investment under multiple time varying demand classes. A hybrid PSO is adopted in order to solve the model. Jiang and Wu (2013) presented a novel approach to solve the conflict between setup frequency and production quantity in an inventory replenishment method based on the theory of constraints supply chain replenishment system (TOC-SCRS) by using PSO and genetic algorithms

Others papers are related to supply chain network design. Bachlaus et al. (2008) attempted to design a multi-echelon supply chain network considering agility as a key design criterion. A novel algorithm entitled hybrid taguchi-particle swarm optimization (HTPSO) was proposed to solve the model. Bashiri and Tabrizi (2010) discussed the application of the theory of constraints into the problem of locating a new distribution center among a producer and a set of existing retailers with random demand. The nonlinear resulting model is solved by a PSO algorithm. Che and Cui (2011) proposed a heuristic-based approach, called GP-TBM, based on a hybrid of the GA and a PSO algorithm by introducing the balance 
modulating (BM) mechanism to solving the mathematical model to find the optimal supply chain network pattern.

Some papers proposed models for reverse supply chain planning. Che et al. (2012) developed an optimization mathematical model solved with PSO in comparison with GA for a reverse supply chain network that contains forward and reverse logistical plans in the multi-echelon system. Che et al. (2014) expanded the model considering fuzzy defect ratio, and fuzzy transport loss ratio. Kannan et al. (2009) designed an integrated forward logistics multi-echelon distribution inventory supply chain model and closed loop multi-echelon distribution inventory supply chain model for the built-to-order environment using GA and PSO. The proposed model is validated in a tire manufacturer and in a plastic goods manufacturer. Chiang et al. (2014) designed a cross-stage reverse logistics course for defective products so that damaged products generated in downstream partners can be directly returned to upstream partners throughout the stages of a supply chain for rework and maintenance. Different variations of PSO algorithms are compared with a GA.

A few works focused on the Vehicle Routing Problems and other variations. See for example Pei et al. (2014), Marinakis et al. (2013 and 2010) and Vahdani et al. (2012). There are few papers in this area because there are other metaheuristics more convenient for this type of problem, such as tabu search among others.

There are some papers associated to partner selection in a supply chain. Zhao et al. (2005) proposed a hybrid algorithm based on PSO and simulated annealing and its applications for partner selection in virtual enterprise and supply chain management. Xu and Yan (2011) focused on the vendor selection problem (VSP) for material supply in large-scale water conservancy and hydropower construction projects, they used PSO to solve this problem in a fuzzy environment. Prasanna proposed a hybrid optimization and simulation approach to design the supply chain sourcing strategy. In the optimization approach, a multiobjective binary particle swarm algorithm is developed for minimizing the total cost and maximizing the supplier delivery reliability. Selected scenarios from the optimization results are modeled using Witness simulation software to evaluate the robustness of sourcing strategies.

Several papers proposed models for the production-distribution problem in a supply chain. Che et al. (2012) and Che et al. (2014) developed a decision methodology for the production and distribution planning of a multi-echelon unbalanced supply chain taking into account such four criteria as cost, quality, delivery and supplier relationship management and considering quantity discount and capacity constraints. The models are solved with PSO and with a methodology based on the analytic network process and turbo particle swarm optimization (TPSO). Jolai et al. (2011) proposed a multiobjective fuzzy goal programming approach with imprecise aspiration levels for integrated production-distribution in a supply chain network to consist of a manufacturer, with multiple plants, products, distribution centers, retailers and customers. PSO algorithm is compared with GA and with a hybrid genetic algorithm. Cárdenas-Barrón and Treviño-Garza (2014) presented a mathematical model for optimizing a three echelon supply chain network. Their model is an integer linear programming model and in order to solve it, they developed five algorithms; four of them are based on a PSO method and the other is a GA. Liu et al. (2012) attempted to realize a distribution network optimization in a supply chain using grey systems theory for uncertain demand. They proposed a hybrid PSO to resolve it.

Finally, others applications based on PSO are in the literature. Gao et al. (2011) established two bi-level programming models for pricing problems with the buyer and the vendor in a supply chain designated as the leader and the follower, respectively. A PSO based algorithm is developed to solve problems defined by these bi-level pricing models. Duran and Perez (2013) presented a model for the Joint Replenishment Problem in a system operating with quantity discounts. As many other works, proposed the definition and the solution of the optimization model using techniques based on the PSO and GA. Huang et al. (2013) studied the coordination of risk management in fashion and textiles supply chain organized as a virtual enterprise (VE). The aim is to find proper decision mechanisms that can improve the overall performance of risk management for the whole $\mathrm{VE}$ as well as each member. The optimization models developed are solved with PSO. Noroozi et al. (2013) proposed a model for the flow shop scheduling problem in a manufacturing supply chain where a group of jobs can be processed on a machine simultaneously. This typical NP-hard problem are solved according three computational intelligence algorithms including a hybrid genetic algorithm, a hybrid simulated annealing and an improved discrete PSO algorithm.

According to the literature, most of the analyzed papers use modified versions of the PSO algorithm in order to solve non-linear or integer linear programming models (both single objective and 
multiobjective). Only a few papers address uncertainty in a supply chain with PSO. Some papers adopting stochastic techniques (Taleizadeh et al. 2013, 2012, 2010; Wong et al. 2009; Yang and Lin, 2010; Bashiri and Tabrizi, 2010) and other apply fuzzy set theory in reverse supply chain management (Che et al. 2014), pricing and lot-sizing (Yaghin et al. 2013), vendor selection (Xu and Yan 2011) and integrated productiondistribution planning (Jolai et al. 2010).

The combination of fuzzy supply chain models and PSO as a solution method is quite recent and the literature review shows that up to our knowledge there is no research that simultaneously deals with supply chain master planning, fuzzy sets theory and PSO applied to a real case. Jolai et al. (2010) proposed an integrated production-distribution problem where fuzzy set theory is used to define the imprecise aspiration levels of goals and not to represent the uncertainty of the model data. Moreover they applied his proposal in a case study.

Therefore, the objectives and contributions of this research are the following:

- To prove the convenience of fuzzy set theory approach and the modified S-curve to model conflicting objectives exist and leads to uncertainty. The hypothesis to be tested and checked is that the fuzzy model $($ alpha $>0)$ should provide better results than the deterministic one (alpha=1).

- To solve the FMOLP model for supply chain planning using the PSO metaheuristics. The only PSO metaheuristics application was proved not to be suitable for managing the accomplishment of all constraints. Therefore, we designed a novel method combining the PSO with a backward calculation, doubled as hybrid PSO-B method. The proposed method provides good quality solutions in a reduced computational time.

The rest of the paper is arranged as follows: Section 2 briefly describes the supply chain configuration from the ceramics sector under consideration. Next, Section 3 refers to the deterministic model and presents the methodology proposed for modeling under uncertainty using the Fuzzy Set Theory, where the incorporation of the method of Torabi and Hassini (2008) is stressed and used to establish an auxiliary simple objective model from an original multi objective fuzzy model. The evaluation of the fuzzy objectives have been made by the application of the modified S-curve transfer function of Vasant et al. (2002). Section 4 presents the solution proposal using a metaheuristics technique (PSO) and provides details about the solution-seeking methodology and the mutation process. In order to avoid the PSO to provide unfeasible solutions, section 5 describes the hybrid PSO-B calculation method proposed. Section 6 presents the main results obtained along with the experimental analysis. Finally, in section 7 the research conclusions and future research lines are exposed.

\section{Description of the ceramics sector supply chain}

The real application of a supply chain initially proposed by Alemany et al. (2010) is taken as the model basis (see Figure 1).

- A multi-plant, multi-line, multi-product and multi-period model. Three distribution levels with multiple distribution centers, logistic centers and shops.

- Replenishment, production and distribution are considered (from the physical perspective). It is assumed that the flow possibilities between the nodes of the various stages, regardless of them being the parts, components, raw materials (RMs), and finished goods or articles (FGs) that circulate through these nodes, have been previously contemplated.

- Subcontracting of finished goods can be partial or total (generally products with low added value).

- FGs are grouped into families. Not all the production lines are capable of processing all the product families; however, the product families that can be processed on each line are known.

- Production within a minimum number of consecutive time periods must be carried out, provided that a production line is ready for a specific product.

[Insert Figure 1 about here] 


\section{Fuzzy mathematical model}

The intention of this section is to solve the supply chain capacitated lot sizing and loading problem (Özdamar and Birbil, 1998), with the model objectives, such as fuzzy variables and solve it by using the PSO metaheuristics. The original NP hard model proposed by Alemany et al. (2010) was transformed into a FMOLP model with fuzzy objectives in Peidro et al. (2012).Therefore, this research work completely adopts this fuzzy model, which is named FMOLP. Readers can consult further details of this model in Peidro et al. (2012).

The methodology proposed by Peidro et al. (2012) is also taken as a basis, where an approach is defined to transform a FMOLP into an equivalent auxiliary crisp mathematical programming model. This approach adopts linear membership functions to represent all the fuzzy objective functions together with the fuzzy programming solution method of Torabi and Hassini (2008). According to Torabi and Hassini (2008), a multi-objective model could be transformed into a single-objective model as follows:

$$
\begin{gathered}
\operatorname{Max} \lambda(x)=\gamma \lambda_{0}+(1-\gamma) \sum_{k} \theta_{k} \mu_{z_{k}}(x) \\
\text { s.t } \lambda_{0} \leq \mu_{z_{k}}(x) \quad k=1, \ldots ., n \\
\quad x \in f(x) \\
\lambda_{0}, \gamma \in[0,1]
\end{gathered}
$$

Where $\mu_{z_{k}}$ and $\lambda_{0}=\min \left\{\mu_{z_{k}}(x)\right\}$ denote the degree of satisfaction of the kth objective function and the minimumdegree of satisfaction of the objectives, respectively. Moreover, $\theta_{k}$ and $\gamma$ indicate the relative importance of the kthobjective function and the compensation coefficient, respectively. The $\theta_{k}$ parameters are determined by the DMbased on his/her preferences so that $\sum_{k} \theta_{k}=1, \theta_{k}>0$.Compensation coefficients determine whether the solution obtained is balanced; in other words, if it gives the same importance to all the objectives, or if it is unbalanced; that is, prioritizes those objectives whose $\theta_{k}$ weight is greater. Any parameter $\gamma$ values close to 1 provide more balanced solutions; otherwise, values close to zero generate solutions with better results for those objectives whose weight is greater. It is the DM's task to determine which type of solutions they wish to obtain when applying the present solution methodology.

The interactive solution procedure adopted from Peidro et al. (2012) is summarized as follows:

Step 1: Formulate the original FMOLP model.

Step 2: Specify the corresponding membership functions for all the fuzzy objectives (upper and lower limits).

Step 3: Determine the corresponding relative importance of the objective functions $\left(\theta_{k}\right)$ and the compensation coefficient $(\gamma)$.

Step 4: Transform the original FMOLP problem into an equivalent single-objective MILP form by using the Torabi and Hassini fuzzy programming method.

Step 5: Solve the proposed auxiliary crisp single-objective model.

Step 6: If the DM is satisfied with this current efficient compromise solution, then stop. Otherwise, go back to Step 2 and provide another efficient solution by changing the controllable parameters value $\left(\theta_{k}\right.$ and $\left.\gamma\right)$.

The only difference between the process described and applied by Peidro et al. (2012) is the fact that they use the linear membership function to describe value $\mu_{z_{k}}(x)$ for each model objective. Since the comparison made in Peidro and Vasant (2011) suggest that the linear membership function can become restrictive in some kind of supply chain problems, it could be better to prove a membership function that is not restrictive as the linear one but flexible enough to describe the vagueness in the fuzzy objectives. Based on that finding, we use the S-curve membership function (Vasant et al. 2002), which is described below (Figure 2):

[Insert Figure 2 about here] 


$$
\mu_{z_{k}}(x)=\left\{\begin{array}{cc}
1 & x<x^{a} \\
0.999 & x=x^{a} \\
\frac{B}{1+C e^{\alpha x}} & x^{a}<x<x^{b} \\
0.001 & x=x^{b} \\
0 & x>x^{a}
\end{array}\right.
$$

The S-curve membership function is a particular case of a logistic function with specific $B, C$ and $\alpha$ values. These values must be described in a heuristic-experimental manner by the decision maker (DM). In this case, the values have been experimentally defined in similar cases with values of $B=1$, $C=0.001001001$ and $\alpha=13.813$. In Equation 2, $\alpha$ determines the degrees of membership of the fuzzy variable in the membership function $\mu(\mathrm{x})$. This value is higher than zero and it represents a measure of the degree of volatility of the study variable; in other words, high $\alpha$ values imply that the fuzzy variable presents increased uncertainty (thus, the solution begins to lose quality the higher this value becomes), whereas if $\alpha$ takes a value of 0 , the tendency of the variable is deterministic. As mentioned earlier, this value must be defined experimentally based on trial and error. We adopt the original values of $B$ and $C$ and estimate values of $\alpha$ that proves experimentally to be better than the proposed with smaller values. Decreasing the alpha sequentially from 13.813 by trial and error, we find a value that has a good performance in the point $\alpha=1.93$, then we decide to maintain this value in the experiments. In order to investigate what happens in an intermediate regions between 1.93 and 0 , we prove as well the scenario $\alpha$ $=0.5$.

Since Peidro et al. (2012) have experimented with the value $\gamma$ and they have found good results with $\gamma=0.1$, we fix this parameter and just vary the alpha value. Meanwhile, the values of the parameter $\theta_{k}$ have been defined in 0.3-0.3-0.4 for the objectives $z 1, z 2$ and $z 3$ respectively, based on DM preferences.

The objectives of the FMOLP model include maximization and minimization. Thus, the modified membership function must take two distinct forms depending on each case. Equation 3 corresponds to the modified S-curve function, adjusted with its $x$ axis for maximization. Equation 4 corresponds to the modified membership function for minimization.

$$
\mu\left(z_{k}\right)=\left\{\begin{array}{cc}
1 & z>z u \\
0.999 & z=z u \\
\frac{B}{1+C e^{\alpha \frac{z u-z}{z u-z l}}} & z l<z<z u \\
0.001 & z=z l \\
0 & z<z l
\end{array} \quad \text { (3) } \quad \mu\left(z_{k}\right)=\left\{\begin{array}{cc}
1 & z<z l \\
0.999 & z=z l \\
\frac{B}{1+C e^{\alpha \frac{z-z l}{z u-z l}}} & z l<z<z u \\
0.001 & z=z u \\
0 & z>z u
\end{array}\right.\right.
$$

In this case, value $z$ corresponds to the result of each fuzzy objective depending on whether it is minimization or maximization. The values $z l$ and $z u$ correspond with the minimum and maximum values that the objective $z$ can take.

\section{Fuzzy model solution using the Particle Swarm Optimization metaheuristic.}

Since each possible solution must be evaluated according to the value of the results in the fuzzy objectives through membership functions, and the auxiliary simple objective model of Torabi and Hassini must be solved with these values, and once again, the application of conventional solvers becomes complex in the iterative process because, among other reasons, the S-curve function evaluation is not easy to model linearly. Another factor that goes against conventional solvers is the problem scale in question, classified as NP Hard, with which complexity, solution time and computational inefficiency can exponentially grow. In line with this, the use of a metaheuristics technique is proposed to obtain good quality solutions in relatively short solution times. The combination of metaheuristics with the fuzzy set theory in the supply chain modeling problems, is a research field that is relatively poorly developed as it was shown in previous literature analysis. In particular, we can see how the most applied types, which have obtained the best results, are population-based heuristic ones and, among them, genetic algorithms are found in particular. Based on this fact, we decide to use a metaheuristics based on population that has achieved good performance in the convergence process according with the literature and that has been little used in combination with fuzzy set theory in supply chain large scale planning problems in order to bring something new to this area. PSO was chosen as the solution method. 


\subsection{Particle Swarm Optimization}

According to Luke (2009), PSO is a method inspired by the interactions of the individuals in a swarm. It does not model after evolution as such, but from a set of interacting individuals, which simulates the swarm's real performance. This method optimizes a problem iteratively in an attempt to improve a candidate solution for a given quality or performance measure. Naturally, as it is a population-based heuristic method, it begins with a set of solutions which function as the initial baseline population, known in this method as the swarm. Solutions are known as particles. The procedure consists in moving the particle across the space of the solution by exploring those sectors where neighboring particles perform better in the problem solution. Exploring is done in accordance with mathematical formulae that indicate the direction and velocity of the search in the solution space.

Unlike other population-based methods, PSO does not re-sample populations to produce new ones; there is no selection of any kind. Instead, it maintains the population static, whose members improve in terms of the response to new discoveries about the space. Essentially, the method is a form of directed mutation and it generally operates in spaces of real values, which reveals a special usefulness for the FMOLP model solution.

\subsubsection{Solution-seeking process}

In the PSO solution procedure, a solution is defined as a vector that contains all the decision variables values. Those values are then used in the problem evaluation in order to find the objective function and the constraints evaluations. This vector in our case is composed by real numbers, defined in determined limits based on the nature and possibilities of each decision variable. Solution vector is known as $\vec{x}$.

In the iterative process, candidate solutions mutate toward the better solutions discovered by the direction and velocity patterns of exploring. Particles never die (no selection exists). Each particle's movement is influenced by its best known local position ("best"), and by the best known position of all the particle population ("gbest"). Accordingly, the whole swarm of particles is expected to move iteratively toward the sector of the space that provides the best solutions. One particle is composed of two parts:

- The particle's location in space $\vec{x}=\left\{x_{1}, x_{2}, \ldots, x_{n}\right\}$. This is the equivalent in evolution algorithms to a chromosome or genome individual.

- Particle velocity $\vec{v}=\left\{v_{1}, v_{2}, \ldots, v_{n}\right\}$. This is the velocity and direction in which the particle is traveling in each iteration.

Each particle begins in a random location and with a random velocity vector. A follow-up must also be done of:

- The best known location $\vec{x}^{*}$ that $\vec{x}$ has discovered.

- The best known location $\vec{x}^{+}$that some $\vec{x}$ informants have discovered. In some PSO algorithm versions, particles are assigned to "network neighbors" who can inform them about the best known locations. Nowadays, $\vec{x}$ informants are small groups of particles selected randomly in each iteration $\vec{x}$ is always one of its own informants.

- The best known location $\vec{x}$ ! that has been discovered by any particle.

The following operations are done in each iteration:

1. Evaluating each particle's performance and updating the best known locations whenever necessary.

2. Determining the mutation mechanism. For each particle $\vec{x}$, its velocity vector $\vec{v}$ is updated by aggregating, to a certain extent, one vector that moves in direction $\vec{x}^{*}$, one vector that moves in direction $\vec{x}$ ! and another vector that moves in direction $\vec{x}$ !. These vectors increase due to uncertainty and randomness noise.

3. Mutating each particle by moving it along its velocity vector.

The PSO algorithm takes the form displayed in Figure 3.

[Insert Figure 3 about here] 


\subsubsection{Mutation}

For the FMOLP model, a mutation operator is required that confers flexibility to the process for real numbers. In order to better handle the resolution process, the binary variables are computed by programming them into the source code depending on the real variables results. Thus we need just a real operator in the mutation process into the PSO algorithm. The mutation operators used in the present study are based on the approach by Srinivas and Deb (1994).

The polynomial mutation operates by selection some position $\vec{x}$. This position is selected with some probability $p$. The number inside the selected position can vary randomly either partially or completely, thus the mutation can imply the change in the complete value of the position of just in some of its decimals. Figure 4 shows an example.

\section{[Insert Figure 4 about here]}

\section{The Hybrid PSO - Backward solution method (PSO-B)}

Often, the size of the problem can make the resolution space so large, causing that metaheuristics cannot find feasible solutions to all the constraints of the model. In our case for example, the PSO can go easily to an infeasible minimum or maximum in the solution space for the balance constraints defined at the different supply chain nodes (equations (17), (24) to (33) of Peidro et al (2012)) due to its huge range of possibilities. To overcome this drawback in large scale supply chain optimization problems, a method that combines the generation lead by the PSO of values for some decision and auxiliary variables (independent) of the problem, that are later used to calculate the value of some other variables (dependent) is proposed. These last dependent variables are calculated by means of the cited equations beginning from the shops to the productive plants in a backward manner. For this reason this procedure is doubled as hybrid PSO-backward solution method.

To apply this method, some auxiliary decision variables that mainly consist of weights belonging to the $[0,1]$ interval have been defined. These weights represent the portion of the input quantity of a destination node that is provided by an origin node. To better understand this concept, assume that a destination node $\mathrm{j}$ is supplied by three origin nodes $i(i 1, i 2, i 3)$ as it is shown in Figure 5 . Therefore, if the global output quantity of a destination node is known (e.g. 1000 units) and a value between $[0,1]$ is given to each of the weights for each origin node (wil, wi2, wi3) in such a manner that all them sum up to one, it is possible to derive the output quantities of each origin node to the destination one by making the corresponding product. Therefore, our calculation method consists of first estimating the value of the global output from a destination node (1000 for our example) and based on it, calculate the input supplied by each origin node based on its assigned weights (wi). This procedure begins at the shop nodes defining the amount of each FG sold in each shop and based on these quantities and the assigned weights to each node, derives the quantity provided by upstream nodes in a backward manner.

\section{[Insert Figure 5 about here]}

Finally, to ensure that the constraints of safety stock of RMs at plants (constraint (5) of Peidro et al. (2012)) and FGs at warehouses (constraint (22) of Peidro et al. (2012)) are respected, the auxiliary variables $E I N A_{i a}, E I N C_{c p t}$, representing the inventory in excess as regards the safety stock are defined. Therefore, the original variables $I N A_{i a}$ and $I N C_{c p t}$ can be calculated as $I N A i a t=E I N A_{i a t}+s s a_{i a}$ and $I N C_{c p t}=$ $E I N C_{c p t}+s s c_{c p}$.

In Figure 6, the hybrid PSO-B solution method is represented. As it can be seen, the variables calculated by the PSO method are: the original decision variables $D I F T K_{i w t}, D I F A_{i a t}, C S C_{i b a t}$, and the auxiliary ones $W C T T K_{\text {iqwit }}, W C T C L_{\text {iaqt }}, W C T A_{\text {ipat }}, W M P_{\text {ilpt }}, E I N A_{\text {iat }}, E I N C_{c p t}$. Then, the original decision variables $V E T K_{i w t}, V E A_{i a t}, M P F_{f l p t}, X_{i l p t}, Y_{f l p t}, Z I_{i l p t}, Z F_{f l p t}$, and $C T P_{\text {crpt }}$ are calculated by means the own equations of the fuzzy model of Peidro et al. (2012); variables $I N A_{\text {iat }}$, and $I N C_{c p t}$ are calculated as shown before from the PSO variables $E I N A_{i a t}$, and $E I N C_{c p t}$, respectively and $C T T K_{\text {iqw }}, C T C L_{\text {iaqt }}, C T A_{\text {ipat }}, M P_{\text {ilpt }}$ variables are calculated based on the weight auxiliary variables $W C T T K_{\text {iqwo }}, W C T C L_{\text {iaqt }}, W C T A_{\text {ipat }}, W M P_{\text {ilpt }}$.

[Insert Figure 6 about here] 


\section{Experimental Analysis.}

The FMOLP model has been programmed in Java and integrated with the JMetal metaheuristics programming framework of Durillo and Nebro (2011). For further details of this metaheuristics programming framework, readers are referred to Durillo and Nebro (2011). It is worth stressing that the PSO algorithm within the jMetal framework has used mainly for unconditional optimization problems, and for those problems in which averaging constraints has been solved, mainly by genetic algorithms. In this case, applying PSO to a problem of this kind is novel enough for this example to set a precedent. Experiments have been ran on a computer with an Intel® Core ${ }^{\mathrm{TM}} 2$ Duo CPU T6670@ $2.20 \mathrm{GHz} \times 2$ processor, with 3GB RAM and in a Linux Ubuntu 14 LTS environment. The input data proposed by Peidro et al (2012) is used to solve the FMOLP model through the hybrid PSO-B solution method with the only exception that we do not consider the minimum lot size and the family setup time. The evaluated alpha values, as it was described earlier, are $\alpha=0, \alpha=0.5$ and $\alpha=1.93$. We use the alpha 0 scenario in order to compare the other two scenarios against it. Making $\alpha=0$ is the same to compare fuzzy scenario versus deterministic conditions. The best $\gamma$ value to prioritize fuzzy objectives with a greater weight is 0.1 (Peidro et al. 2012). These experiments should lead to determinate at first the computation efficiency of the PSO-B method in large scale problems. Then we aim to prove the convenience of applying fuzzy set theory in this kind of problem versus just consider the deterministic scenario in terms of the objective function values.

The limits set for fuzzy objectives are the same used by Peidro et al. ( 2012):

- $\quad z 1$ (Total profit): between 200000 and 370000

- $\quad z 2$ (Backorder/Delayed demand): between 0 and 5920

- $\quad z 3$ (Idle time in the system): between 0 and 620

Finally, the jMetal framework in its PSO algorithm does not itself consider the constraints are fulfilled because the algorithm is used mainly to solve unconditioned models. The methodology to integrate a constraint fulfillment parameter into the PSO algorithm is to use a penalization factor. In experimental terms, it is verified that the best method to penalize the objective function in our case is to consider a constraint fulfillment factor; in this case, the objective function is penalized by subtracting an exponential accumulated percentage of constraints that breach the rule, as shown below:

$$
\operatorname{Max} \lambda(x)=\left(\gamma \lambda_{0}+(1-\gamma) \sum_{k} \theta_{k} \mu_{z_{k}}\right) * e^{\left(50 *\left(\frac{S R}{T R}-1\right)\right)}
$$

Where the SR (surplus constraints) factor refers to the quantity of constraints that are breached, whereas the TR factor refers to all the evaluated constraints. The factor 50 in the exponential is just a very big number that guarantees that the objective function is strongly penalized as the breached constraints go up. The penalty factor goes to 1 as the breached constraints lower. Table 1 summarizes the main results and the comparison among each alpha scenario evaluated:

\section{[Insert Table 1 about here]}

The execution was made by setting a total size of 250 to the initial swarm population. It has been considered a total of 1000 iterations; then the execution time reported is the exact time in which PSO finds the best solution through the mutation process. Then, it can be seen how the convergence process is very fast. The rest of the iterations the mutation process searches for better solutions, but if it does not find some new, it maintains that considered as the Gbest of the mutation process.

The results of the membership function for the alpha 0 scenario do not appear in table 1 because it is the deterministic scenario. The resolution of the alpha 0 scenario is the same to take the original model of Alemany et al. (2010) and solve it without considering minimum lot size and family setups.

It can be observed how the objective function value is very close to 1 in each of the fuzzy cases, being both of them better than deterministic case. When each objective is separately analyzed, it can be highlighted that, judging by the global objective function, the alpha 0.5 scenario is better because it is the scenario with best results in the backorder quantity generated and the total idle time. Meanwhile, the scenario with alpha 1.93 is the best if the judging is based on the total benefit generated. The final 
selection between these two possibilities depends on the preferences of decision-maker taking into account the risk level of each one and the final preference of each of the three objectives. In general terms, we can conclude that both of them achieve very good results in the objective function, and we find out how our method is capable to handle with the uncertainty generated by conflictive objectives and how our proposed calculation method PSO-B achieves very good results in terms of computational efficiency.

PSO-B method and the fuzzy set theory have proven at first that both fuzzy scenarios outperforms the deterministic one, mainly in the total backorder generated and the total benefit. But, the main findings are that fuzzy approach is able to handle the available inventories and production capacity in a better way than the deterministic conditions, what supports the hypothesis that fuzzy set theory can improve the general performance in supply chain problems that are affected for some uncertainty in the objectives or where it exists a conflictive situation among objectives like minimize the production cost but at the same time to minimize the idle time of the production lines what is our case. Meanwhile, our second main finding is how the computational efficiency proves that the method PSO-B permits the handling of a large scale problem combined with the fuzzy set theory without sacrifice a considerable quantity of additional execution time. In fact, it can be notice how the execution time of each one of the three scenarios in the experiments is very little. This means that the backward calculation is able to strongly reduce the resolution complexity in a problem like this.

\section{Conclusions}

This paper continues the work of Peidro et al. (2012) that proposes a FMOLP to solve the master planning problem for the replenishment-production-distribution of ceramic supply chains for the purpose of maximizing the total gross margin, minimizing backorder quantities, and minimizing the idle production time in multi-supplier, multi-plant, multi-type, multi-level distribution centers with a multi-item and multi-period logistic environment.

In this paper, the convenience of applying the fuzzy set theory where conflicting objectives exist, has been proved. In presence of conflictive objectives, where a good result in one can imply a worse result in the other, it is suggested the application of fuzzy set theory in order to better handle the uncertainty generated by this conflict. The modified S-curve membership function (Peidro and Vasant, 2011) works very well in the description of fuzzy objectives in this kind of supply chain optimization problems.

The FMOLP model belongs to the CLSLP with backlogging and setup carry-over from which little literature can be found (Karimi et al. 2003). Since these problems are NP-hard, fast and efficient heuristics are required. Metaheuristics can be used to solve this kind of problems because their main importance is to find good solutions to a very hard problem in an execution time relatively short. In this paper we propose the PSO metaheuristics to efficiently solve the problem. Up to our knowledge there are not papers dealing simultaneously with supply chain planning, fuzzy and PSO. The only PSO application to solve this kind of problem often leads to unfeasible solutions where some constraints related to material balance equations at supply chain nodes and the relationship among binary and continuous variables representing setups are violated. For this reason, we have proposed a good performing procedure called backward calculation that consist in finding feasible solutions beginning from values (provided by the PSO) of the decision variables implied at the most downstream supply chain nodes (shops in our case) and following the calculation in a backward direction through the supply chain upstream nodes.

The combination of the PSO with backward calculation, doubled as hybrid PSO-B, has proved a very good performance in the resolution and convergence process. We have found good solutions in a very short execution time. The hybrid PSO-B method has been applied to solve the deterministic and uncertainty version of the FMOLP model. Results are better when considering uncertainty, proving that the fuzzy approach is able to handle resources capacity and inventory better than the deterministic one. This proves the convenience of the application of fuzzy set theory what was exactly one of our objectives. Furthermore the hybrid PSO-B method has been shown to be able to handle large size problems in a fuzzy environment in a reduced computational time.

Finally among possible future research lines, evaluating other bio inspired metaheuristics techniques can be considered, such as ant colonies or genetic algorithms, to determine whether performance in such problems outperforms PSO. Furthermore, the proposed method can be applied to models that include technologically fuzzy constraints and coefficients. 


\section{Acknowledgements}

This research is partly supported by the Spanish Ministry of Economy and Competitiveness projects: "Methods and models for operations planning and order management in supply chains characterized by uncertainty in production due to the lack of product uniformity" (PLANGES-FHP) (Ref. DPI2011-23597) and "Operations design and Management of Global Supply Chains" (GLOBOP) (Ref. DPI2012-38061C02-01); and by the project funded by the Polytechnic University of Valencia entitled 'Quantitative Models for the Design of Socially Responsible Supply Chains under Uncertainty Conditions. Application of Solution Strategies based on Hybrid Metaheuristics' (PAID-06-12).

\section{References}

Alemany, M.M.E., Boj, J.J., Mula, J., Lario, F.-C., 2010. Mathematical programming model for centralised master planning in ceramic tile supply chains. Int. J. Prod. Res. 48, 5053-5074. doi:10.1080/00207540903055701

Bachlaus, M., Pandey, M.K., Mahajan, C., Shankar, R., Tiwari, M.K., 2008. Designing an integrated multi-echelon agile supply chain network: a hybrid taguchi-particle swarm optimization approach. J. Intell. Manuf. 19, 747-761. doi:10.1007/s10845-008-0125-1

Bashiri, M., Tabrizi, M.M., 2010. Supply chain design: A holistic approach. Expert Syst. Appl. 37, 688693. doi:10.1016/j.eswa.2009.06.006

Cárdenas-Barrón, L.E., Treviño-Garza, G., 2014. An optimal solution to a three echelon supply chain network with multi-product and multi-period. Appl. Math. Model. 38, 1911-1918. doi:10.1016/j.apm.2013.09.010

Che, Z., Chiang, T.-A., Che, Z.-G., 2012. Using analytic network process and turbo particle swarm optimization algorithm for non-balanced supply chain planning considering supplier relationship management. Trans. Inst. Meas. Control 34, 720-735. doi:10.1177/0142331211402901

Che, Z.H., Chiang, T.-A., Kuo, Y.C., Cui, Z., 2014. Hybrid Algorithms for Fuzzy Reverse Supply Chain Network Design. Sci. World J. 2014, 1-16. doi:10.1155/2014/497109

Che, Z.H., Cui, Z., 2011. Unbalanced supply chain design using the analytic network process and a hybrid heuristic-based algorithm with balance modulating mechanism. Int. J. Bio-Inspired Comput. 3, 56. doi:10.1504/IJBIC.2011.038703

Chiang, T.-A., Che, Z.H., Cui, Z., 2014. Designing a Multistage Supply Chain in Cross-Stage Reverse Logistics Environments: Application of Particle Swarm Optimization Algorithms. Sci. World J. 2014, 1-19. doi:10.1155/2014/595902

Duran, O., Perez, L., 2013. Solution of the Spare Parts Joint Replenishment Problem with Quantity Discounts Using a Discrete Particle Swarm Optimization Technique. Stud. Inform. CONTROL 22, 319-328.

Durillo, J.J., Nebro, A.J., 2011. jMetal: A Java framework for multi-objective optimization. Adv. Eng. Softw. 42, 760-771. doi:10.1016/j.advengsoft.2011.05.014

Gao, Y., Zhang, G., Lu, J., Wee, H.-M., 2011. Particle swarm optimization for bi-level pricing problems in supply chains. J. Glob. Optim. 51, 245-254. doi:10.1007/s10898-010-9595-8

Ghasemy Yaghin, R., Fatemi Ghomi, S.M.T., Torabi, S.A., 2013. A possibilistic multiple objective pricing and lot-sizing model with multiple demand classes. Theme Optim. Games 231, 26-44. doi:10.1016/j.fss.2012.11.012

Huang, M., Wang, X., Lu, F.-Q., Bi, H.-L., 2013. A Coordination of Risk Management for Supply Chains Organized as Virtual Enterprises. Math. Probl. Eng. 2013, 1-11. doi:10.1155/2013/931690

Jiang, X.., Wu, H.., 2013. Optimization of setup frequency for TOC supply chain replenishment system with capacity constraints. Neural Comput. Appl. 23 1831-1838.

Jolai, F., Razmi, J., Rostami, N.K.M., 2011. A fuzzy goal programming and meta heuristic algorithms for solving integrated production: distribution planning problem. Cent. Eur. J. Oper. Res. 19, 547569. doi:10.1007/s10100-010-0144-9

Kannan, G., Haq, A.N., Devika, M., 2009. Analysis of closed loop supply chain using genetic algorithm and particle swarm optimisation. Int. J. Prod. Res. 47, 1175-1200. doi:10.1080/00207540701543585 
Karimi, B., Fatemi Ghomi, S.M.T., Wilson, J.M., 2003. The capacitated lot sizing problem: a review of models and algorithms. Omega 31, 365-378. doi:10.1016/S0305-0483(03)00059-8

Kennedy, J., Eberhart, R.., 1995. Particle swarm optimization. Int. Conf. Neural Netw. Vol IVIEEE Serv. Cent. Piscataway NJ 1942-1948.

Liu, S., Forrest, J., Yangjie, Y., Zhang, K., Mi, C., Xie, N., Zhang, Q., Wang, H., Liu, H., 2012. 4-stage distribution network optimization of supply chain with grey demands. Kybernetes 41, 633-642.

Luke, S., 2009. Essentials of metaheuristics. Lulu Raleigh.

Marinakis, Y., Iordanidou, G.-R., Marinaki, M., 2013. Particle Swarm Optimization for the Vehicle Routing Problem with Stochastic Demands. Appl. Soft Comput. 13, 1693-1704. doi:10.1016/j.asoc.2013.01.007

Marinakis, Y., Marinaki, M., Dounias, G., 2010. A hybrid particle swarm optimization algorithm for the vehicle routing problem. Eng. Appl. Artif. Intell. 23, 463-472. doi:10.1016/j.engappai.2010.02.002

Mousavi, S.M., Hajipour, V., Niaki, S.T.A., Aalikar, N., 2014. A multi-product multi-period inventory control problem under inflation and discount: a parameter-tuned particle swarm optimization algorithm. Int. J. Adv. Manuf. Technol. 70, 1739-1756. doi:10.1007/s00170-013-5378-y

Noroozi, A., Mokhtari, H., Kamal Abadi, I.N., 2013. Research on computational intelligence algorithms with adaptive learning approach for scheduling problems with batch processing machines. Neurocomputing 101, 190-203. doi:10.1016/j.neucom.2012.08.011

Özdamar, L., Birbil, Ş.İ., 1998. Hybrid heuristics for the capacitated lot sizing and loading problem with setup times and overtime decisions. Eur. J. Oper. Res. 110, 525-547. doi:10.1016/S03772217(97)00269-5

Park, K., Kyung, G., 2014. Optimization of total inventory cost and order fill rate in a supply chain using PSO. Int. J. Adv. Manuf. Technol. 70, 1533-1541. doi:10.1007/s00170-013-5399-6

Peidro, D., Mula, J., Alemany, M.M.E., Lario, F.-C., 2012. Fuzzy multi-objective optimisation for master planning in a ceramic supply chain. Int. J. Prod. Res. 50, 3011-3020. doi:10.1080/00207543.2011.588267

Peidro, D., Vasant, P., 2011. Transportation planning with modified S-curve membership functions using an interactive fuzzy multi-objective approach. Appl. Soft Comput. 11, 2656-2663. doi:10.1016/j.asoc.2010.10.014

Pei, J., Liu, X., Pardalos, P.M., Fan, W., Yang, S., Wang, L., 2014. Application of an effective modified gravitational search algorithm for the coordinated scheduling problem in a two-stage supply chain. Int. J. Adv. Manuf. Technol. 70, 335-348. doi:10.1007/s00170-013-5263-8

PrasannaVenkatesan, S., Kumanan, S., 2012. Multi-objective supply chain sourcing strategy design under risk using PSO and simulation. Int. J. Adv. Manuf. Technol. 61, 325-337. doi:10.1007/s00170011-3710-y

Sadeghi, J., Mousavi, S.M., Niaki, S.T.A., Sadeghi, S., 2013. Optimizing a multi-vendor multi-retailer vendor managed inventory problem: Two tuned meta-heuristic algorithms. Knowl.-Based Syst. 50, 159-170. doi:10.1016/j.knosys.2013.06.006

Sadeghi, J., Sadeghi, S., Niaki, S.T.A., 2014. Optimizing a hybrid vendor-managed inventory and transportation problem with fuzzy demand: An improved particle swarm optimization algorithm. Inf. Sci. 272, 126-144. doi:10.1016/j.ins.2014.02.075

Srinivas, N., Deb, K., 1994. Muiltiobjective Optimization Using Nondominated Sorting in Genetic Algorithms. Evol. Comput. 2, 221-248. doi:10.1162/evco.1994.2.3.221

Sue-Ann, G., Ponnambalam, S.G., Jawahar, N., 2012. Evolutionary algorithms for optimal operating parameters of vendor managed inventory systems in a two-echelon supply chain. Adv. Eng. Softw. 52, 47-54. doi:10.1016/j.advengsoft.2012.06.003

Taleizadeh, A.A., Niaki, S.T.A., Makui, A., 2012. Multiproduct multiple-buyer single-vendor supply chain problem with stochastic demand, variable lead-time, and multi-chance constraint. Expert Syst. Appl. 39, 5338-5348. doi:10.1016/j.eswa.2011.11.001

Taleizadeh, A.A., Niaki, S.T.A., Shafii, N., Meibodi, R.G., Jabbarzadeh, A., 2010. A particle swarm optimization approach for constraint joint single buyer-single vendor inventory problem with changeable lead time and (r,Q) policy in supply chain. Int. J. Adv. Manuf. Technol. 51, 12091223. doi:10.1007/s00170-010-2689-0

Taleizadeh, A.A., Niaki, S.T.A., Wee, H.-M., 2013. Joint single vendor-single buyer supply chain problem with stochastic demand and fuzzy lead-time. Knowl.-Based Syst. 48, 1-9. doi:10.1016/j.knosys.2013.03.011 
Torabi, S.A., Hassini, E., 2008. An interactive possibilistic programming approach for multiple objective supply chain master planning. Theme Games Decis. 159, 193-214. doi:10.1016/j.fss.2007.08.010

Vahdani, B., Tavakkoli-Moghaddam, R., Zandieh, M., Razmi, J., 2012. Vehicle routing scheduling using an enhanced hybrid optimization approach. J. Intell. Manuf. 23, 759-774. doi:10.1007/s10845010-0427-y

Vasant, P., Nagarajan, R., Yaacob, S., 2002. Decision making using modified S-curve membership function in fuzzy linear programming problem. J. Inf. Commun. Technol.

Wong, J.-T., Chen, K.-H., Su, C.-T., 2009. Replenishment decision support system based on modified particle swarm optimization in a VMI supply chain. Int. J. Ind. Eng. Theory Appl. Pract. Vol 16 No 12009.

$\mathrm{Xu}$, J., Yan, F., 2011. A multi-objective decision making model for the vendor selection problem in a bifuzzy environment. EXPERT Syst. Appl. 38, 9684-9695. doi:10.1016/j.eswa.2011.01.168

Yang, M.-F., Lin, Y., 2010. Applying the linear particle swarm optimization to a serial multi-echelon inventory model. EXPERT Syst. Appl. 37, 2599-2608. doi:10.1016/j.eswa.2009.08.021

Zhao, F., Zhang, Q., Yu, D., Chen, X., Yang, Y., 2005. A Hybrid Algorithm Based on PSO and Simulated Annealing and Its Applications for Partner Selection in Virtual Enterprise, in: Huang, D.-S., Zhang, X.-P., Huang, G.-B. (Eds.), Advances in Intelligent Computing. Springer Berlin Heidelberg, Berlin, Heidelberg, pp. 380-389.

\section{Biographical notes}

Hanzel Grillo Espinoza is a Ph.D. student at the Universitat Politècnica de València (UPV) in Spain. He is an industrial engineer graduated from University of Costa Rica and cursed the Master in Advanced Engineering of Production, Logistics and Supply Chain at the UPV. He is now working in the Research Centre on Production Management and Engineering (CIGIP) with a specialization scholarship, participating in Spanish and European projects while developing his doctoral Ph.D. in the area of mathematical modeling of order management process affected by uncertainties, making use of techniques like fuzzy set theory and metaheuristics. His research work and interest areas are focused precisely on mathematical modeling and optimization, simulation, quality control production and logistics. Before the starting in the doctoral process, he has worked in multinational companies like Procter\&Gamble and Walmart Stores in the Central America region developing projects of efficiency improving in operative process in stores, logistic centers and human resources handling.

Dr. David Peidró Payá is an Associate Professor in Operations Management and Operations Research at the Universitat Politècnica de València (UPV) in Spain. He is an industrial engineer and received her PhD in Industrial Engineering from the UPV. He is member of Research Centre on Production Management and Engineering (CIGIP) and has participated in several Spanish and European Projects. His research is focused on Fuzzy Supply Chain Planning, Application of Soft Computing to Transport Planning and Sustainable Supply Chain Management. He has several research papers in books, conferences and international journals with high impact such as FSS, IJPE, IJPR, ASC, EJOR, PP\&C and others.

Dr. Ma Mar Eva Alemany Díaz is an Associate Professor in Operations Management and Operations Research at the Universitat Politècnica de València (UPV) in Spain. She is an industrial engineer and received her $\mathrm{PhD}$ in Industrial Engineering from the UPV. She is member of Research Centre on Production Management and Engineering (CIGIP) and has participated in several Spanish and European Projects. Currently, she is leading a Spanish Government Project. Her research is focused on Hierarchical Production Planning, Collaborative Order Promising Process and Supply Chain Management under conceptual and quantitative perspective. She has several research papers in books, conferences and international journals with high impact such as IJPE, COMIND, AMM, IJPR, SIC, PP\&C and others.

Dr. Josefa Mula Bru is a Senior Lecturer in operations research and supply chain simulation at the Universitat Politècnica de València. She is member of the scientific committee of the Research Centre on Production Management and Engineering (CIGIP) in which she has developed R\&D Projects in the areas of industrial management and supply chain management. Her key research topics include production planning and control, supply chain management and uncertainty modelling. She has published (in collaboration) more than sixty research papers in a number of leading journals. She has written (in 
collaboration) the books Supply Chain Simulation: A System Dynamics Approach for Improving Performance (2011) and Operations Research Problems: Statements and Solutions (2013). She is editor of the Journal of Industrial Engineering and Management, International Journal of Production Management and Engineering, Journal of Industrial Engineering, The Scientific World Journal and Industrial Engineering and Management.

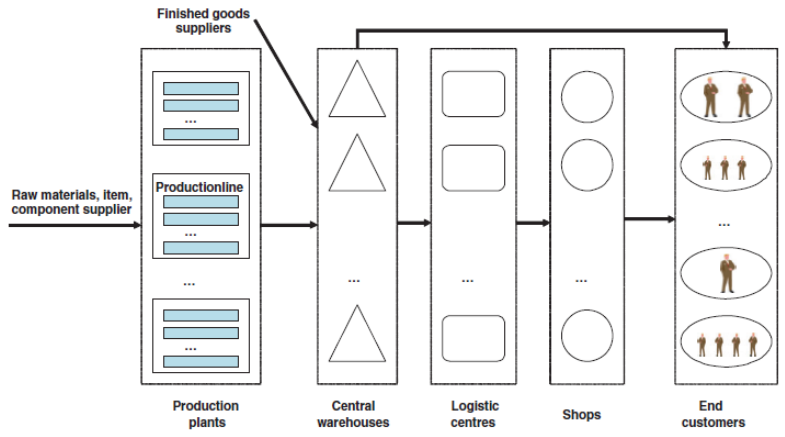

Figure 1 Supply chain distribution for the practical case applied (Alemany et al. 2010)

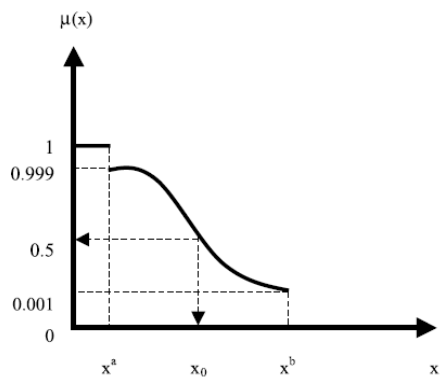

Figure 2 S-curve membership function (Vasant et al. 2002)

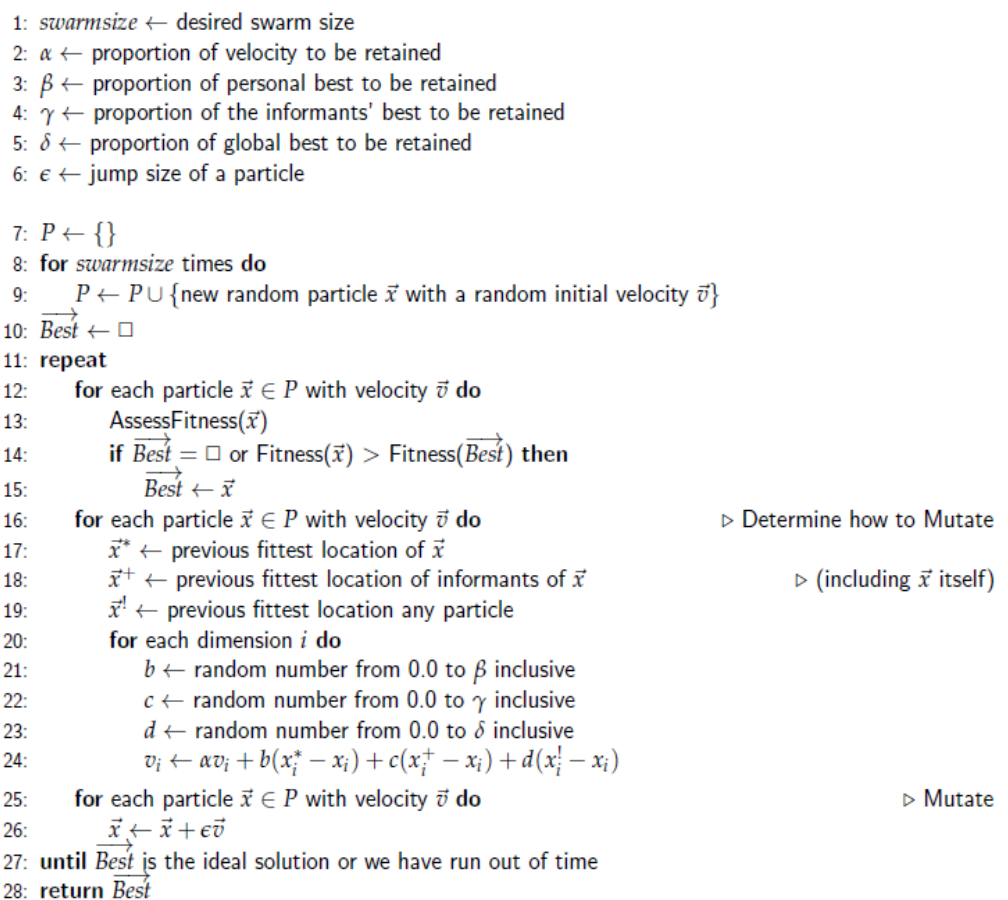

Figure 3 Structure of PSO (Luke, 2009) 
Int. J., Vol. x, No. x, 200x

\section{Solution vector $\vec{x}$}

Initial solution

\begin{tabular}{|l|l|l|l|l|}
\hline 12.234 & 15.769 & 23.456 & 8.235 & 16.346 \\
\hline
\end{tabular}

Element to mutate with probability $\mathrm{p}$

\begin{tabular}{|l|l|l|l|l|}
\hline 12.234 & 15.769 & $\mathbf{2 3 . 4 5 6}$ & 8.235 & 16.346 \\
\hline
\end{tabular}

New solution

\begin{tabular}{|l|l|l|l|l|}
\hline 12.234 & 15.769 & 23.389 & 8.235 & 16.346 \\
\hline
\end{tabular}

Figure 4 Polynomial mutation



Figure 5 Backward calculation 


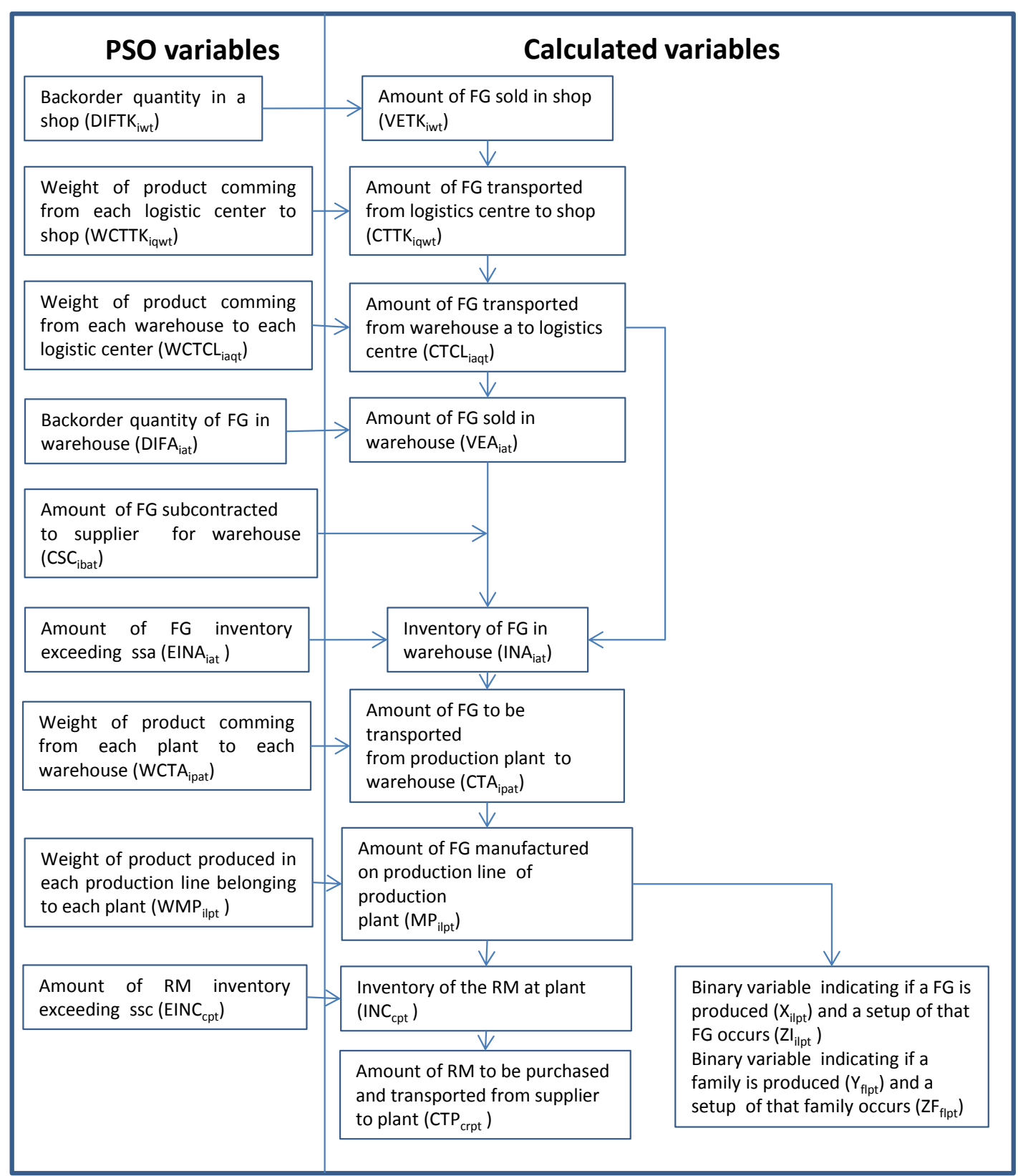

Figure 6 Hybrid PSO-B method global overview 
Int. J., Vol. x, No. x, 200x

Table 1 Objective function results and computational efficiency

\begin{tabular}{|c|c|c|c|c|c|}
\hline \multicolumn{6}{|c|}{ Objective results } \\
\hline \multirow[b]{2}{*}{ Objectives } & \multirow[b]{2}{*}{$\alpha=\mathbf{0}$} & \multicolumn{2}{|c|}{$\alpha=0.5$} & \multicolumn{2}{|c|}{$\alpha=1.93$} \\
\hline & & PSO-B & $\begin{array}{c}\% \text { dif vs alpha } 0 \\
\text { scenario }\end{array}$ & PSO-B & $\begin{array}{c}\% \text { dif vs alpha } 0 \\
\text { scenario }\end{array}$ \\
\hline Obj. (Z1): Total benefit & $458,715.5$ & $463,000.9$ & $0.9 \%$ & $471,169.4$ & $2.7 \%$ \\
\hline Obj. (Z2): Backorder & $5,076.3$ & $4,327.5$ & $-14.8 \%$ & $4,708.4$ & $-7.2 \%$ \\
\hline Obj. (Z3): Idle time & 510.1 & 492.0 & $-3.5 \%$ & 521.4 & $2.2 \%$ \\
\hline$\mu\left(\mathrm{Z}_{1}\right)$ & & 1.0 & & 1.0 & \\
\hline$\mu\left(\mathrm{Z}_{2}\right)$ & & 0.998 & & 0.996 & \\
\hline$\mu\left(\mathrm{Z}_{3}\right)$ & & 0.998 & & 0.995 & \\
\hline Objective function & & 0.999 & & 0.998 & \\
\hline \multicolumn{6}{|c|}{ Computational efficiency } \\
\hline Real variables PSO-B & $1,476.0$ & $1,476.0$ & $0.0 \%$ & $1,476.0$ & $0.0 \%$ \\
\hline Total constraints & $2,832.0$ & $2,832.0$ & $0.0 \%$ & $2,832.0$ & $0.0 \%$ \\
\hline Execution time(s) & 5.30 & 5.10 & $-2.1 \%$ & 6.10 & $16.0 \%$ \\
\hline
\end{tabular}

